\title{
Patient Radiation Exposure During Diagnostic and Therapeutic Procedures for Intracranial Aneurysms: A Multicenter Study
}

\author{
Yon Kwon Ihn, MD', Bum-Soo Kim, MD², Jun Soo Byun, MD³, Sang Hyun Suh, MD4, \\ Yoo Dong Won, MD5, Deok Hee Lee, MD' , Byung Moon Kim, MD7, Young Soo Kim, MD, \\ Pyong Jeon, MD', Chang-Woo Ryu, MD'0, Sang-il Suh, MD", Dae Seob Choi, MD'2, \\ See Sung Choi, MD'13, Jin Wook Choi, MD'4, Hyuk Won Chang, MD'5, Jae-Wook Lee, MD' ${ }^{16}$, \\ Sang Heum Kim, MD ${ }^{17}$, Young Jun Lee, MD ${ }^{18}$, Shang Hun Shin, MD', Soo Mee Lim, MD ${ }^{20}$, \\ Woong Yoon, MD ${ }^{21}$, Hae Woong Jeong, MD'22, Moon Hee Han, MD ${ }^{23}$
}

Purpose: To assess patient radiation doses during cerebral angiography and embolization of intracranial aneurysms across multi-centers and propose a diagnostic reference level (DRL).

\footnotetext{
'Department of Radiology, St.Vincent's Hospital, College of Medicine, The Catholic University of Korea, Gyeonggi-do, Korea ${ }^{2}$ Department of Radiology, Seoul St. Mary's Hospital, College of Medicine, The Catholic University of Korea, Seoul, Korea ${ }^{3}$ Department of Radiology, Chung-Ang University Hospital, Seoul, Korea

${ }^{4}$ Department of Radiology, Gangnam Severance Hospital, Yonsei University, Seoul, Korea

${ }^{5}$ Department of Radiology, Uijeongbu St. Mary's Hospital, College of Medicine, The Catholic University of Korea, Gyeonggi-do, Korea ${ }^{6}$ Department of Radiology and Research Institute of Radiology, University of Ulsan College of Medicine, Asan Medical Center, Seoul, Korea ${ }^{7}$ Department of Radiology, Severance Hospital Stroke Center, Yonsei Unviersity College of Medicine, Seoul, Korea ${ }^{8}$ Department of Neurosurgery, Pusan National University Yangsan Hospital, Pusan National University, Yangsan, Korea פDepartment of Radiology, Samsung Medical Center, Sungkyunkwan University School of Medicine, Seoul, Korea ${ }^{10}$ Department of Radiology, Kyung Hee University Hospital at Gangdong, Seoul, Korea

${ }^{11}$ Department of Radiology, Korea University Guro Hospital, Korea University College of Medicine, Seoul, Korea

${ }^{12}$ Department of Radiology, Gyeongsang National University Hospital, Gyeongsang National University School of Medicine, Jinju, Korea

${ }^{13}$ Department of Radiology, Wonkwang University Hospital, Iksan, Korea

${ }^{14}$ Department of Radiology, Ajou University School of Medicine, Gyeonggi-do, Korea

${ }^{15}$ Department of Radiology, Keimyung University College of Medicine, Dongsan Medical Center, Daegu, Korea

${ }^{16}$ Department of Radiology, National Health Insurance Service Ilsan Hospital, Gyeonggi-do, Korea

${ }^{17 D e p a r t m e n t ~ o f ~ R a d i o l o g y, ~ C H A ~ B u n d a n g ~ M e d i c a l ~ C e n t e r, ~ C H A ~ U n i v e r s i t y, ~ G y e o n g g i-d o, ~ K o r e a ~}$

${ }^{18}$ Department of Radiology, Hanyang University College of Medicine, Seoul, Korea

${ }^{19}$ Department of Radiology, Ulsan University Hospital, University of Ulsan, College of Medicine, Ulsan, Korea

${ }^{20}$ Department of Radiology, Ewha Womans University Mokdong Hospital, Seoul, Korea

${ }^{21}$ Department of Radiology, Chonnam National University Hospital, Gwangju, Korea

${ }^{22}$ Department of Radiology, Busan Paik Hospital, Inje University, Busan, Korea

${ }^{23}$ Department of Radiology, Seoul National University Hospital, Seoul National University College of Medicine, Seoul, Korea

Received April 19, 2016; accepted after revision August 11, 2016.
}

Correspondence to: Bum-Soo Kim, MD, Department of Radiology, Seoul St. Mary's Hospital, College of Medicine, The Catholic University of Korea, 222 Banpo-daero, Seocho-gu, Seoul 06591, Korea.

Tel. 82.2.2258.6239 Fax. 82.2.599.6771 E-mail: bumrad@catholic.ac.kr

This is an Open Access article distributed under the terms of the Creative Commons Attribution Non-Commercial License (http://creativecommons.org/licenses/by-nc/3.0) which permits unrestricted non-commercial use, distribution, and reproduction in any medium, provided the original work is properly cited. 
Materials and Methods: We studied a sample of 490 diagnostic and 371 therapeutic procedures for intracranial aneurysms, which were performed at 23 hospitals in Korea in 2015. Parameters including dose-area product (DAP), cumulative air kerma (CAK), fluoroscopic time and total angiographic image frames were obtained and analyzed.

Results: Total mean DAP, CAK, fluoroscopy time, and total angiographic image frames were $106.2 \pm$ $66.4 \mathrm{~Gy}-\mathrm{cm}^{2}, 697.1 \pm 473.7 \mathrm{mGy}, 9.7 \pm 6.5$ minutes, $241.5 \pm 116.6$ frames for diagnostic procedures, $218.8 \pm 164.3 \mathrm{~Gy}-\mathrm{cm}^{2}, 3365.7 \pm 2205.8 \mathrm{mGy}, 51.5 \pm 31.1$ minutes, $443.5 \pm 270.7$ frames for therapeutic procedures, respectively. For diagnostic procedure, the third quartiles for DRLs were 144.2 Gy-cm² for DAP, 921.1 mGy for CAK, 12.2 minutes for fluoroscopy times and 286.5 for number of image frames, respectively. For therapeutic procedures, the third quartiles for DRLs were 271.0 Gy-cm² for DAP, $4471.3 \mathrm{mGy}$ for CAK, 64.7 minutes for fluoroscopy times and 567.3 for number of image frames, respectively. On average, rotational angiography was used $1.5 \pm 0.7$ times/session (range, $0-4 ; n=490$ ) for diagnostic procedures and $1.6 \pm 1.2$ times/session (range, $0-4 ; n=368$ ) for therapeutic procedures, respectively.

Conclusion: Radiation dose as measured by DAP, fluoroscopy time and image frames were lower in our patients compared to another study regarding cerebral angiography, and DAP was lower with fewer angiographic image frames for therapeutic procedures. Proposed DRLs can be used for quality assurance and patient safety in diagnostic and therapeutic procedures.

Key Words : Cerebral angiography; Cerebral embolization; Diagnostic reference levels; Radiation dose

Cerebrovascular disease is one of the leading causes of death in Korea, and there has been a corresponding increase in the number and complexity of interventional neuroradiology (INR) procedures. This increase is attributable to both technological advancements and evidence favoring endovascular techniques over conventional surgery [1]. However, interventional radiological procedures have the potential to exposure a patient to high doses of radiation, and, in particular, a high entrance surface dose of up to several Gy. Surface skin dose is important because of the potential for welldocumented deterministic effects, such as skin epilation, erythema and desquamation. Dose thresholds for these lesions are in the order of $3 \mathrm{~Gy}, 6 \mathrm{~Gy}$ and 15 Gy, respectively [2]. Additionally, INR procedures, like all sources of radiation exposure, can cause stochastic risks by way of cancer. Direct measurement of surface skin dose is desirable, but not practical because thermoluminescent dosimeters (TLD) are cumbersome with limitations in terms of practical use [3]. An indirect estimate of the radiation dose using a dose-area product (DAP) meter, which is common in modern angiographic systems, can be an alternative.

A diagnostic reference level (DRL) is an investigational level used to identify unusually high radiation doses for common diagnostic medical X-ray imaging procedures. DRLs are suggested action levels above which a facility should review its method and determine if an acceptable image quality can be achieved at lower doses [4]. It is typically set as the 75 th percentile of measured patient or phantom data. Commonly used parameters for a DRL dose are dose area product (DAP), number of exposures and fluoroscopy time.

Previous studies in Korea and other countries have investigated DRL levels, and reported a large variation within hospitals using different DRL guidelines [5-7]. Recognizing the need for continuous monitoring of the dose in INR procedures and the fact that no such multicenter study has ever been carried out in Korea so far, the authors have collected data concerning the dose to patients undergoing some of the most common diagnostic and therapeutic INR procedures performed in Korea.

The purposes of present study were: (1) to obtain baseline data by evaluation of patient exposure at multicenters, (2) to compare these data with the recent literature from other studies, and (3) to propose DRLs.

\section{MATERIALS AND METHODS}

This study was approved by our Institutional Review Board. A waiver of the need for consent was obtained for the Health Insurance Portability and Accountability 
Act compliant survey research.

The study included patients undergoing INR procedures from October 2015 through to December 2015. Twenty-three university hospitals in Korea were invited to participate in the study. Each center was asked to register 20 diagnostic and 15 therapeutic INR procedures. As the radiation doses show a wide distribution with respect to pathology and type of INR procedure, we focused on a more standardized examination, such as diagnostic cerebral angiography and therapeutic embolization for an intracranial aneurysm. For each examination, the centers were requested to fill out a questionnaire containing various information regarding radiation data. The X-ray systems used in this study were all biplane DSA (15 Siemens, 7 Philips and 1 GE machines.).

\section{Procedures included}

The INR procedures were divided into two groups: first, diagnostic cerebral angiography for aneurysm evaluation; and second, therapeutic procedures, namely aneurysmal coil embolization. Diagnostic procedures performed for follow-up after clipping or coiling of an aneurysm were excluded. The procedures were performed by an experienced interventional neuroradiologist or a clinical fellow undergoing interventional neuroradiology training, all using their own protocols.

\section{Data collection}

The patient's demographic information was excluded for each procedure. Collected patient radiation dose indicators were as follows: DAP $\left(\mathrm{Gy}-\mathrm{cm}^{2}\right)$, cumulative air kerma (CAK), fluoroscopic exposure time, number of angiographic image acquisitions. Collected data from AP and lateral views were added together and compared for analysis. The number of rotational angiographic acquisitions in each session of diagnostic and therapeutic procedures was also surveyed. Data were analysed to assess mean \pm standard deviation for each parameter. As collected radiation doses showed a skewed distribution with extreme values and a long upper tail, 75th percentile values were also analysed to propose DRLs.

\section{RESULTS}

A total of 861 patients were included in this survey, with 490 diagnostic and 371 therapeutic procedures. The results, including the third quartiles, are presented in Table 1. In diagnostic cerebral angiography, mean $\mathrm{DAP} \pm$ standard deviation were $106.2 \pm 66.4 \mathrm{~Gy}-\mathrm{cm}^{2}$ for DAP, $697.1 \pm 473.7 \mathrm{mGy}$ for CAK, $9.7 \pm 6.5$ minutes for fluoroscopic time, and $241.5 \pm 116.6$ frames for total angiographic image frames. For therapeutic procedures, mean DAP \pm standard deviation was $218.8 \pm 164.3 \mathrm{~Gy}-\mathrm{cm}^{2}$ for DAP, 3365.7 $\pm 2205.8 \mathrm{mGy}$ for CAK, $51.5 \pm 31.1$ minutes for fluoroscopic time, and $443.5 \pm 270.7$ frames for total angiographic image frames. The third quartiles, which may be set as a DRL were 144.2 and $271.0 \mathrm{~Gy}-\mathrm{cm}^{2}$ for DAP, 921.1 and $4471.3 \mathrm{mGy}$ for cumulative air kerma, 12.2 and 64.7 minutes for fluoroscopy times and 286.5 and 567.3 for the number of image frames in diagnostic and therapeutic procedures, respectively. The distribution plots of the two types of INR procedures in all hospitals are shown in Figure 1 and 2. On average, rotational angiography was used $1.5 \pm 0.7$ times/ session (range, $0-4 ; n=490$ ) for diagnostic procedures and $1.6 \pm 1.2$ times/session (range, $0-4 ; n=368$ ) for therapeutic procedures, respectively.

Table 1. Mean and Third Quartile Data for DAP, Cumulative Air Kerma and Image Frames for Diagnostic and Therapeutic Procedure

\begin{tabular}{|c|c|c|c|c|c|c|}
\hline & Sample size & & $\mathrm{DAP}\left(\mathrm{Gy}-\mathrm{cm}^{2}\right)$ & $\begin{array}{l}\text { Cumulative Air } \\
\text { Kerma (mGy) }\end{array}$ & $\begin{array}{c}\text { Fluoroscopy } \\
\text { time (min) }\end{array}$ & $\begin{array}{c}\text { Number of } \\
\text { image frames }\end{array}$ \\
\hline $\begin{array}{l}\text { Diagnostic cerebral } \\
\text { angiography }\end{array}$ & 490 patients & $\begin{array}{l}\text { Mean } \pm S D \\
\text { Median } \\
\text { 3rd quartile } \\
\text { Range } \\
\text { 25th percentile }\end{array}$ & $\begin{array}{c}106.2 \pm 66.4 \\
89.4 \\
144.2 \\
15.5-468.0 \\
54.9\end{array}$ & $\begin{array}{c}697.1 \pm 473.7 \\
511.3 \\
921.1 \\
102.2-3058.0 \\
362.0\end{array}$ & $\begin{array}{c}9.8 \pm 6.5 \\
7.3 \\
12.2 \\
1.1-42.4 \\
12.2\end{array}$ & $\begin{array}{c}241.5 \pm 116.6 \\
228.5 \\
286.5 \\
52-844 \\
155.0\end{array}$ \\
\hline $\begin{array}{l}\text { Interventional } \\
\text { cerebral } \\
\text { embolization for } \\
\text { aneurysm }\end{array}$ & 371 patients & $\begin{array}{l}\text { Mean } \pm \text { SD } \\
\text { Median } \\
\text { 3rd quartile } \\
\text { Range } \\
\text { 25th percentile }\end{array}$ & $\begin{array}{c}218.8 \pm 164.3 \\
179.0 \\
271.0 \\
20.0-1154.4 \\
104.5\end{array}$ & $\begin{array}{c}3365.7 \pm 2205.8 \\
2804.0 \\
4471.3 \\
486.9-11256.0 \\
1691.8\end{array}$ & $\begin{array}{c}51.5 \pm 31.1 \\
44.5 \\
64.7 \\
4.9-251.8 \\
30.4\end{array}$ & $\begin{array}{c}443.5 \pm 270.7 \\
412.5 \\
567.3 \\
57-1441 \\
241.5\end{array}$ \\
\hline
\end{tabular}

Note.- DAP $=$ dose-area product 


\section{DISCUSSION}

Dose-area product (DAP) action levels are defined as levels of radiation that indicate skin exposures necessitating medical follow-up for possible radiation injuries. DAP action levels do not provide a guideline for optimizing the dose in relation to both medical benefit and risk of injury. However, DRLs do. Comparing the local values with proposed DRLs gives an idea of how the current practice in a hospital ranks with respect to
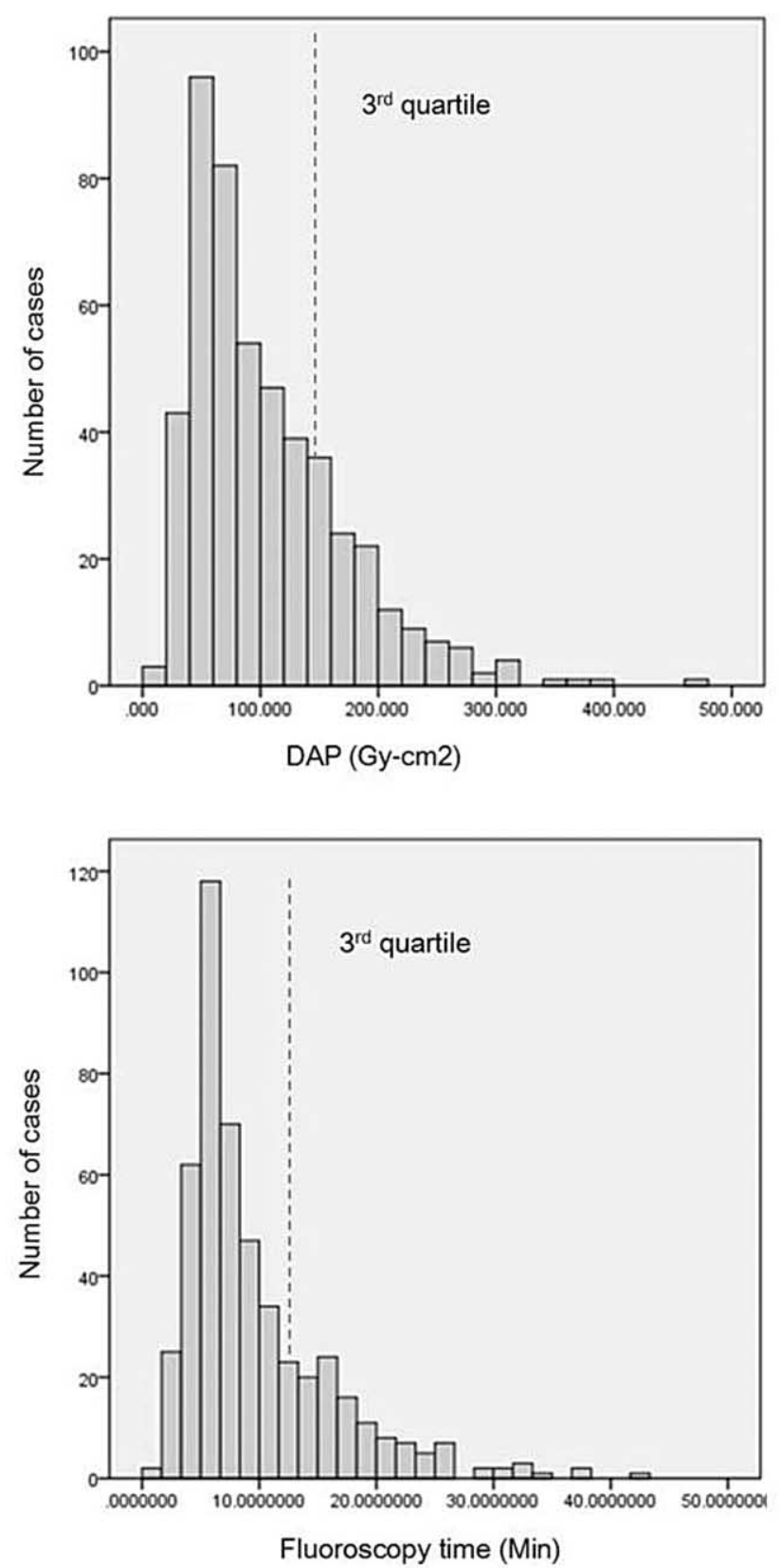

the patient's dose [8].

Tables 2 and 3 present comparisons between our results and those published in the literature [6,7,9-11]. With 861 total cases, our study is the largest to date regarding cerebral aneurysms for a proposed DRL. For cerebral angiographic procedures associated with aneurysms, mean DAP, fluoroscopy time and number of angiographic frames were lower in our patients than in the other studies. For cerebral embolization procedures, our data showed comparatively lower DAP, longer fluoroscopy times, and fewer angiographic
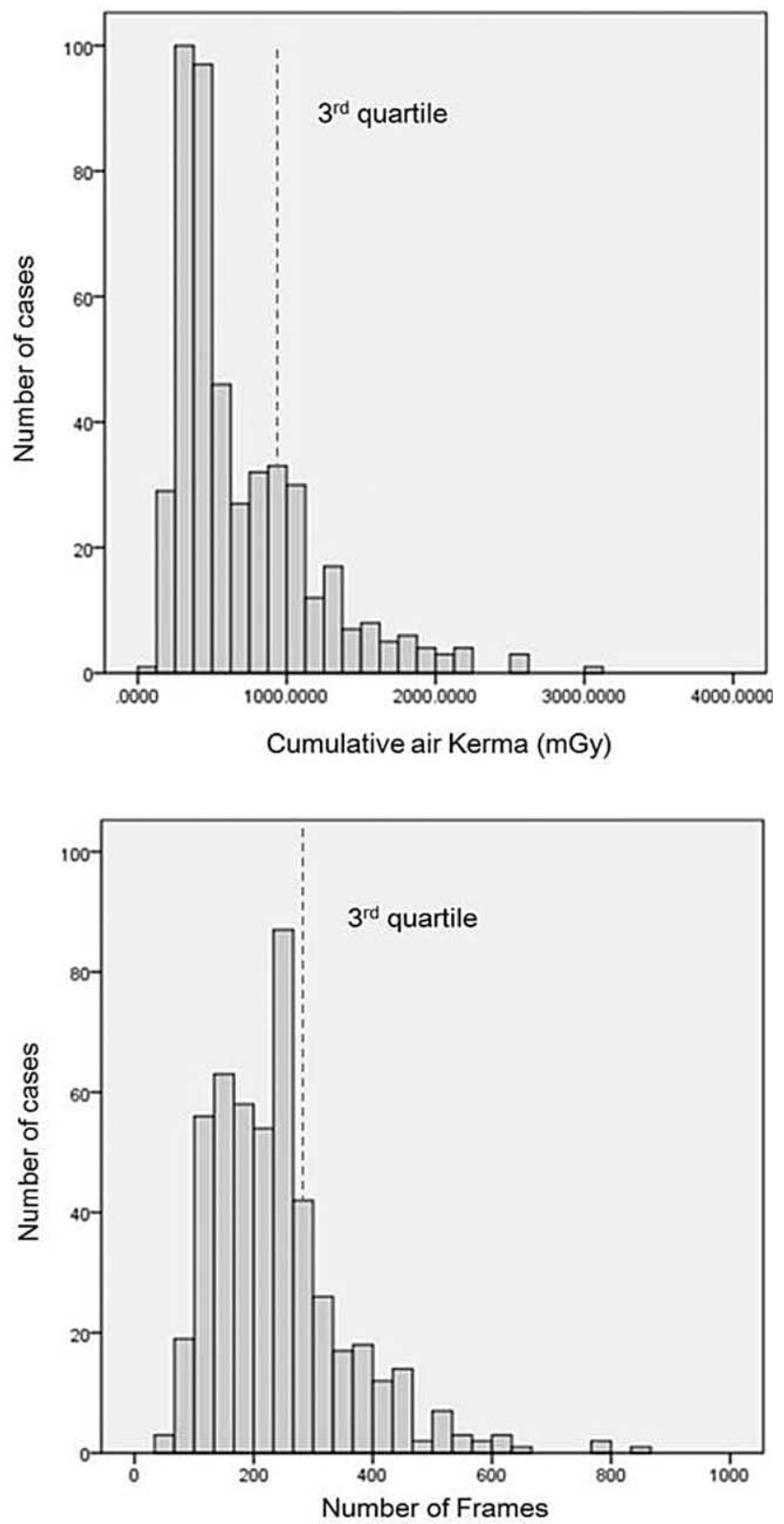

Fig. 1. Distribution of the DAP, cumulative air kerma, fluoroscopy time and number of frames for diagnostic procedures. Dashed lines represent third quartile. 
image frames compared to previous reports. The RADIR study [11] reports mean DAP values of $320 \mathrm{~Gy}-\mathrm{cm}^{2}$ for 382 cases, including embolization of aneurysm, AVM and tumors. Vano et al. [14] reports a mean DAP of $305 \mathrm{~Gy}-\mathrm{cm}^{2}$ and median DAP value of $256 \mathrm{~Gy}-\mathrm{cm}^{2}$ for 172 embolization procedures. However, D'Ercole et al. [9] reports a higher median value of $352 \mathrm{~Gy}-\mathrm{cm}^{2}$ for 82 embolization procedures.

The results on skin doses are difficult to compare with other studies because different metrics have been used. Some authors report peak skin dose, measured or
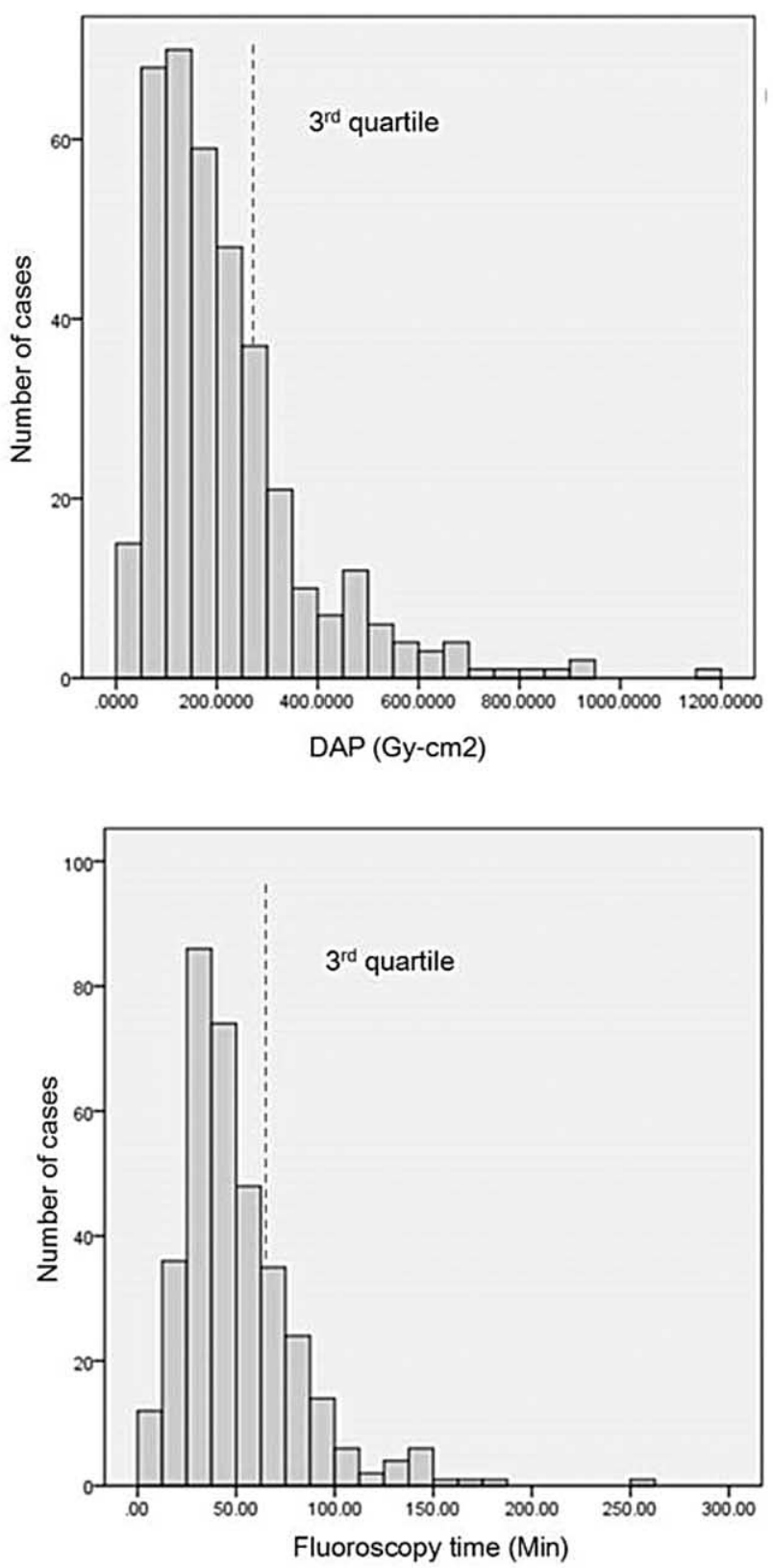

estimated [3, 9, 11-13], whereas we report DAP and reference air kerma as displayed by the angiography system. The mean skin absorbed doses in our study were 697.1 and $3365.7 \mathrm{mGy}$ in diagnostic and therapeutic procedures, respectively. The RAD-IR study [11] also reports mean CAK values of $3.8 \mathrm{~Gy}$ for interventional neuroradiology procedures with aneurysms and AVMs. The study by Vano et al. [14] reported a CAK mean of $2.7 \mathrm{~Gy}$ and a CAK third quartile value of $3.9 \mathrm{~Gy}$ for cerebral embolizations. Our value of mean CAK 3.4 Gy was within the range of
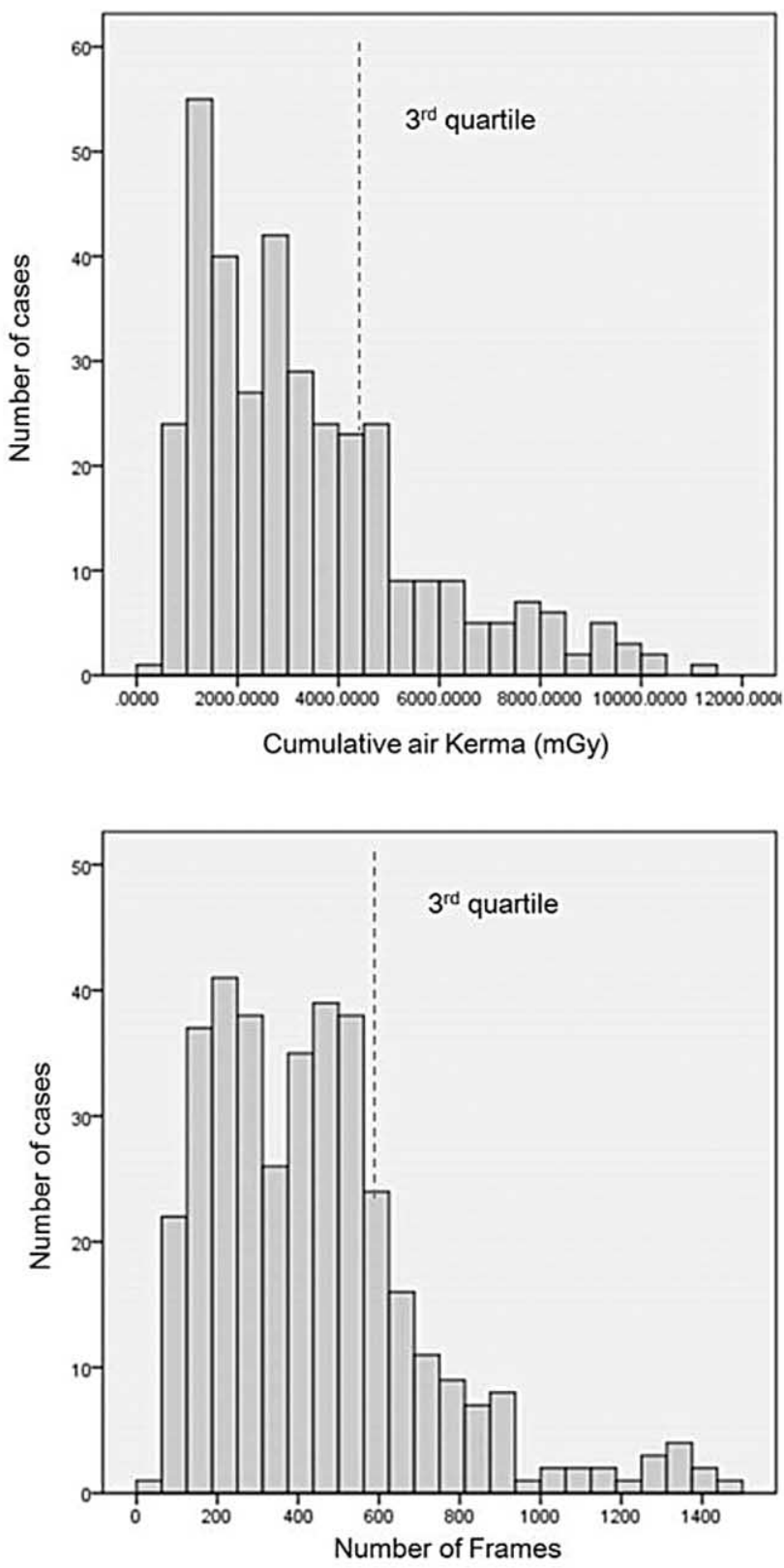

Fig. 2. Distribution of the DAP, cumulative air kerma, fluoroscopy time and number of frames for aneurysm embolization. Dashed lines represent third quartile. 
previous published reports, and in concordance with the data in previously published reports, this suggests that our findings provide at least approximate applicability to other hospitals. The term "cumulative skin dose," introduced by ICRP (International Commission on Radiologic Protection), is used in this article as the value of the dose in air, accumulated at the entrance of the patient during the whole interventional procedure. It is obtained from the air kerma accumulated at a specific point in space relative to the fluoroscopic gantry. The "cumulative skin dose" is not the same quantity as the "peak skin dose (PSD)" (i.e., the highest dose on any portion of a patient's skin during a procedure) [14]. PSD measurement is the best indicator for assessing the likelihood of deterministic effects. Unfortunately, PSD measurement is complex and this capability is not yet widely available. Cumulative skin dose is a reasonable alternative [11]. The ICRP has recommended that maximum cumulative absorbed doses to the skin approaching or exceeding $1 \mathrm{~Gy}$ (for procedures that may be repeated) or 3 Gy (for any other procedure) should be recorded in the patient's record and that there should be follow-up procedures for such cases [15].
From figure 3, we observed large dose variations for the procedures between different hospitals, even within the same procedure type. As an example, mean DAPs range from between 51.9 and $247.6 \mathrm{~Gy}-\mathrm{cm}^{2}$ and fluoroscopy times range from between 3.5 to 18.6 minutes for diagnostic angiography. This is caused by inter-institutional differences in procedure complexity, study protocol, equipment, practitioner skill and technique. Struelens et al. [16] suggested that a large dose variation is caused by a difference in the number of frames, number of projections and exposure parameters. Furthermore, Neofotistou et al. [17] attributed higher doses to teaching hospitals in their study, as it is known that in their first year of training, operators use higher levels of radiation due to extended fluoroscopy.

Regarding the diagnosis and treatment of cerebral aneurysms, rotational angiography facilitates evaluation of the potential for endovascular treatment, permits finding a good working view, aids in performing accurate measurements, depicts additional aneurysms, enables accurate determination of stent positions during stent-assisted coiling, and evaluates subarachnoid hemorrhage during the therapeutic procedure [18-20]. Our study showed that rotational angiography was used

Table 2. Review of Mean and Third Quartile Data of DAP, Fluoroscopic Time and Number of Image Frames Acquired During Diagnostic Cerebral Angiography for Intracranial Aneurysms

\begin{tabular}{|c|c|c|c|c|c|c|c|}
\hline \multirow{2}{*}{ Reference } & \multirow{2}{*}{ No. of Patients } & \multicolumn{2}{|c|}{$\mathrm{DAP}\left(\mathrm{Gy}-\mathrm{cm}^{2}\right)$} & \multicolumn{2}{|c|}{ Fluoroscopy Time (Min) } & \multicolumn{2}{|c|}{ Number of Frames } \\
\hline & & Mean & 3rd Quartile & Mean & 3rd Quartile & Mean & 3rd Quartile \\
\hline This study & 490 & 106.2 & 144 & 9.8 & 12.2 & 242 & 287 \\
\hline Aroua et al.(7) & 91 & 121 & 125 & 12.6 & 15 & 679 & 480 \\
\hline D’Ercole et al.(9) & 100 & 142.1 & 180 & 9.9 & 12.3 & 220 & 317 \\
\hline Brambilla et al.(10) & 188 & 158 & 198 & 13.7 & 17.5 & & \\
\hline Chun et al.(6) & 439 & 136.6 & 154.2 & 12.6 & 14.0 & 251 & 273 \\
\hline
\end{tabular}

Note.- DAP $=$ dose-area product

Table 3. Review of Mean and Third Quartile Data of DAP, Fluoroscopic Time and Number of Image Frames Acquired During Coil Embolization of Intracranial Aneurysms

\begin{tabular}{|c|c|c|c|c|c|c|c|}
\hline \multirow{2}{*}{ Reference } & \multirow{2}{*}{ No. of Patients } & \multicolumn{2}{|c|}{$\mathrm{DAP}\left(\mathrm{Gy}-\mathrm{cm}^{2}\right)$} & \multicolumn{2}{|c|}{ Fluoroscopy Time (Min) } & \multicolumn{2}{|c|}{ Number of Frames } \\
\hline & & Mean & 3rd Quartile & Mean & 3rd Quartile & Mean & 3rd Quartile \\
\hline This study & 371 & 218.8 & 271 & 51.5 & 64.7 & 444 & 567 \\
\hline Aroua et al.(7) & 52 & 335 & 440 & 36.5 & 50 & 760 & 800 \\
\hline D’Ercole et al.(9) & 72 & 382.2 & 487 & 37.2 & 46.3 & 558 & 717 \\
\hline Chun et al.(6) & 111 & 226.0 & 272.8 & 52.9 & 61.1 & 241 & 276 \\
\hline Miller et al.(11) & 356 & 319.9 & & 87.1 & & 1053 & \\
\hline
\end{tabular}

Note.- DAP = dose-area product 

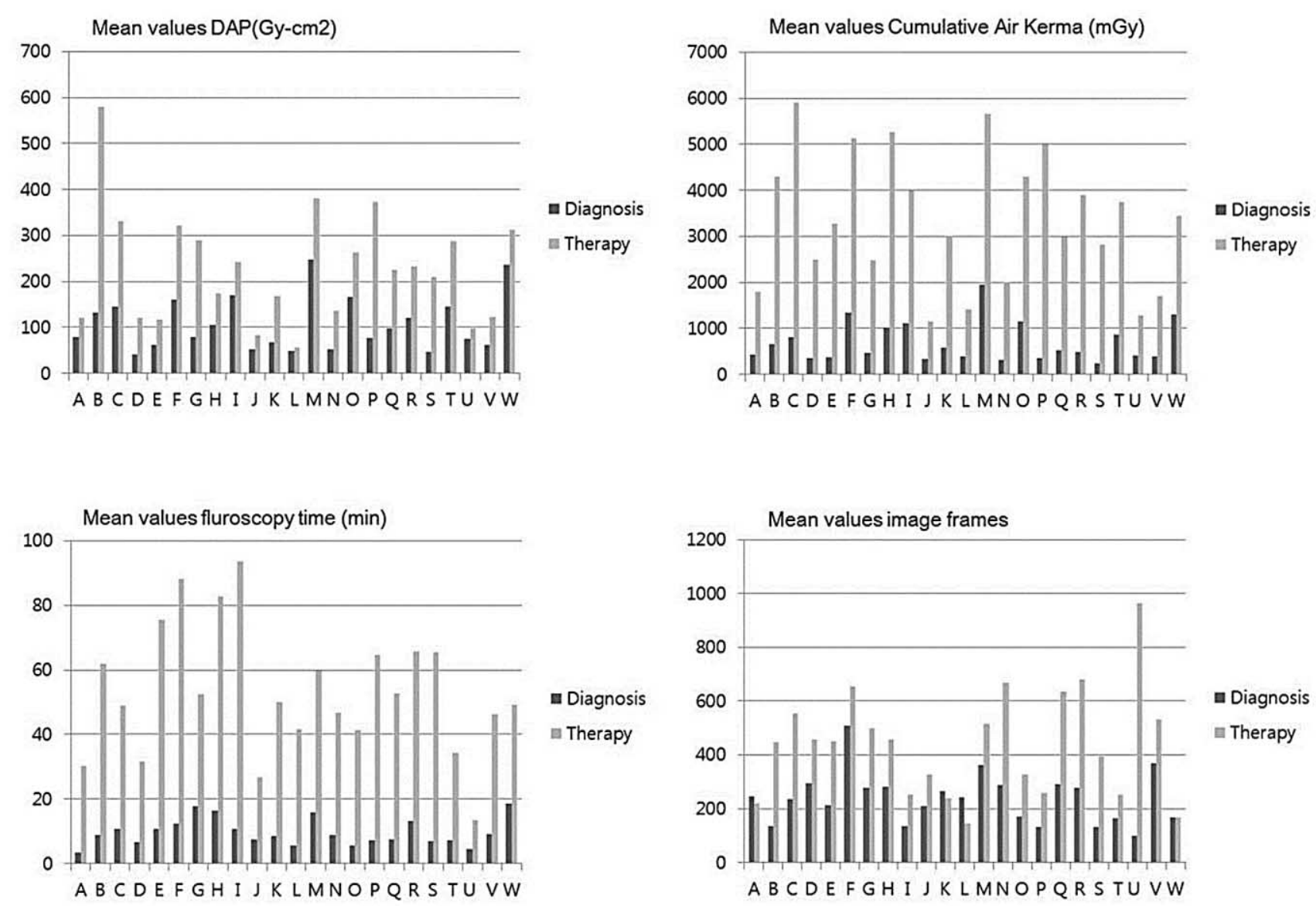

Fig. 3. Comparison of the mean values of the DAP, cumulative air kerma, fluoroscopy time and number of frames for diagnostic and therapeutic procedures for multi-centers. The alphabet of $x$-axis represents the various hospitals.

more frequently in therapeutic procedures than diagnostic angiography (1.63 vs. 1.45 times/session), likely due to complexity of the procedure.

Setting up DRLs for dose-intensive examinations involving fluoroscopy is a difficult task due to the large variability of the fluoroscopic time and the number of images leading to a wide distribution of patient doses. This is due to several factors, including the often loose definition of the examination, differences in the techniques and protocol used, the variability in the complexity of the cases and the experience of the radiologist [7]. DRLs cannot be used individually on a patient basis and are not a restrictive threshold, but do provide a practical guideline for monitoring and comparing radiation doses among procedures and institutions [8].

Unique institutional patterns and operator preferences for INR procedures can cause difficulty in making radiation dose comparisons between hospitals. Nevertheless, this study would indicate that most Korean university hospitals are delivering appropriate levels of radiation to patients during INR procedures. Our proposed DRL would be of value in comparing and monitoring radiation dose. There are a wide variety of INR procedures with varying complexity which are continuously progressing, so radiation dose may be higher with complex, newer or meticulous procedures. Nevertheless, it is each practitioner's responsibility to investigate his or her own practice and to limit unnecessary radiation exposure according to the ALARA (As Low as Reasonably Achievable) principle [21].

This study has limitations. First, we reviewed a prospectively collected database of diagnostic cerebral angiograms. Inclusion criteria included consecutive patients undergoing diagnostic cerebral angiography from September to December in 2015. We did not evaluate the complexity of the proceudures. Duration of fluoroscopic exposure for each procedure was strongly dependent on the individual clinical circumstances. Second, we selected institutions at which INR procedures were performed frequently, which might have biased the results. Further investigation with 
larger populations and prospective evaluation is clearly warranted in order to validate our results.

\section{CONCLUSIONS}

In the present study, radiation dose as measured by DAP, fluoroscopy time and image frames were lower in our patients than in other studies involving cerebral angiography, and DAP was lower with fewer angiographic image frames regarding aneurysm embolization. We proposed $144 \mathrm{~Gy}-\mathrm{cm}^{2}$ and $271 \mathrm{~Gy}-$ $\mathrm{cm}^{2}$ as DRLs for diagnostic and therapeutic procedures, respectively. A national registry of radiation dose is a necessary next step to refine the reference level.

\section{Acknowledgements}

This study was supported by the Korean Society of Interventional Neuroradiology (KSIN) research grant. The authors wish to thank the nurses, technical personnel and staff of interventional neuroradiology at all hospitals involved in this study for their participation and their help with dose measurements.

\section{References}

1. Molyneux AJ, Kerr RS, Yu LM, Clarke M, Sneade M, Yarnold JA, et al. Interventional subarachnoid aneurysm trial(ISAT) of neurosurgical clipping versus endovascular coiling in 2143 patients with ruptured intracranial aneurysms: a randomized comparison of effects on survival, dependency, seizures, rebleeding, subgroups, and aneurysm occlusion. Lancet 2005;366:809 817

2. Norbash AM, Busick D, Marks MP. Techniques for reducing interventional neuroradiologic skin dose: tube position rotation and supplemental beam filtration. AJNR Am J Neuroradiol 1996;17:41-49

3. Suzuki S, Furui S, Matsumaru Y, Nobuyuki S, Ebara M, Abe T, et al. Patient skin dose during neuroembolization by multiple-point measurement using a radiosensitive indicator. AJNR Am J Neuroradiol 2008;29:1076-1081

4. ICRP 103: the 2007 Recommendations of the International Commission on Radiological Protection. Ann ICRP 2007;37(2-4): $1-332$

5. Chung JW; Korea Food \& Drug Administration. Evaluation of patient dose in interventional radiology. Seoul: Korea Food \& Drug Administration; 2007

6. Chun CW, Kim BS, Lee CH, Ihn YK, Shin YS. Patient radiation dose in diagnostic and interventional procedures for intracranial aneurysms: experience at a single center. Korean J Radiol 2014; $15: 844-849$

7. Aroua A, Rickli H, Stauffer JC, Schnyder P, Trueb PR, Valley JF, et al. How to set up and apply reference levels in fluoroscopy at a national level. Eur Radiol 2007;17:1621-1633

8. Bogaert E, Bacher K, Lemmens K, Carlier M, Desmet W, De Wagter X, et al. A large-scale multicentre study of patient skin doses in interventional cardiology; dose-area product action levels and dose reference levels. Br J Radiol 2009;82:303-312

9. D'Ercole L, Thyrion FZ, Bocchiola M, Mantovani L, Klersy C. Proposed local diagnostic reference levels in angiography and interventional neuroradiology and a preliminary analysis according to the complexity of procedures. Phys Med 2012;28:61-70

10. Brambilla M, Marano G, Dominietto M, Cotroneo AR, CarrieroA. Patient radiation doses and references levels in interventional radiology. Radiol Med 2004;107:408-418

11. Miller DL, Balter S, Cole PE, Lu HT, Schueler BA, Geisinger M, et al. Radiation doses in interventional radiology procedures: the RAD-IR study: part I: overall measurement of dose. J Vasc Interv Radiol 2003;14:711-727

12. Theodorakou $\mathrm{C}$, Horrocks JA. A study on radiation doses and irradiated areas in cerebral embolization. Br J Radiol 2003;76: 546-552

13. D'Ercole L, Mantovani L, Thyrion FZ, Bocchiola M, Azzaretti A, Di Maria F, et al. A study on maximum skin dose in cerebral embolization procedures. AJNR Am J Neuroradiol 2007;28:503507

14. Vano E, Fernandez JM, Sanchez RM, Martinez D, Ibor LL, Gil A, et al. Patient radiation dose management in the follow-up of potential skin injuries in neuroradiology. AJNR Am J Neuroradiol 2013;34:277-282

15. Valentin J. Avoidance of radiation injuries from medical interventional procedures. Ann ICRP 2000;30:7-67

16. Struelens L, Vanhavere F, Bosmans H, Van Loon R, Mol H. Skin dose measurements on patients for diagnostic and interventional neuroradiology: a multicenter study. Radiat Prot Dosimetry 2005;114:143-146

17. Neofotistou V, Vano E, Padovani R, Kotre J, Dowling A, Toivonen M, et al. Preliminary reference levels in interventional cardiology. Eur Radiol 2003;13:2259-2263

18. van Rooij WJ, Sprengers ME, de Gast AN, Peluso JP, Sluzewski M. 3D rotational angiography: the new gold standard in the detection of additional intracranial aneurysms. AJNR Am J Neuroradiol 2008;29:976-979

19. Richter G, Engelhorn T, Stuffert T, Doelken M, Ganslandt O, Hornegger J, et al. Flat panel detector angiographic CT for stentassisted coil embolization of broad-based cerebral aneurysms. ANJR Am J Neuroradiol 2007;28:1902-1908

20. Doelken M, Struffert T, Richter G, Engelhorn T, Nimsky C, Ganglandt O, et al. Flat-panel detector volumetric CT for visualization of subarachnoid hemorrhage and ventricles: preliminary results compared to conventional CT. Neuroradiology 2008;50: 517-523

21. Alexander MD, Oliff MC, Olorunsola OG, Brus-Ramer M, Nickoloff EL, Meyers PM. Patient radiation exposure during diagnostic and therapeutic interventional neuroradiology procedures. J Neurointerv Surg 2010;2:6-10 